motions. Conclusion: Participants were engaged in the activity and had positive reaction to the SAR. Further studies are needed to a) test the robot in real world settings and b) over longer time periods.

\section{REMOTE, SMARTPHONE APP-BASED ASSESSMENT OF NORMAL AND DUAL TASK WALKING IN HEALTHY YOUNGER AND OLDER ADULTS} W. Yu ${ }^{1}$, H. Zhu ${ }^{2}$, O. Lo $^{3}$, J. Zhou ${ }^{4}$, R. Harrison ${ }^{2}$, L. Lipsitz ${ }^{5}$, A. Pascual-Leone ${ }^{5}$, B. Manor ${ }^{6}, 1$. Institute for Aging Research, Hebrew SeniorLife, Boston, MA, USA, 2. Hebrew SeniorLife Institute for Aging Research, 3. Institute for Aging Research, Hebrew Senior Life, Boston, MA, USA, 4. Harvard Medical School/Hebrew SeniorLife, 5. Harvard Medical School, 6. Harvard Medical SchooNHebrew SeniorLife Institute for Aging Research

The ability to walk under normal and cognitive dual task conditions is essential for safe mobility in older adults. However, while assessments of gait provide important health information, they have been limited to laboratory settings. We aimed to create an easy-to-use smartphone App enabling remote dual task walking assessments, and to demonstrate its reliability and validity in relatively healthy younger and older adults. The iPhone-based App utilized multi-media instructions and the phone's internal motion sensors to record movements during normal walking and walking with simultaneous serial-subtractions, with the phone placed in the user's pants pocket. Fourteen younger and 12 older adults completed two lab visits, during which data were acquired by the App and GAITRite mat over multiple normal and dual task walking trials. Participants also used the App to complete assessments in their homes on three separate days. Outcomes included average stride time and the dual-task cost to stride time. Across all detected strides in laboratory trials, derived outcome measures from the App were highly correlated with those derived from the GAITRite mat (Stride Time: $\mathrm{p}<0.001, \mathrm{r} 2=0.99$. Dual-task cost: $\mathrm{p}<0.001, \mathrm{r} 2=0.98)$. These correlations were unaffected by age group, walking condition, and pocket tightness. Moreover, across trials, gait metrics demonstrated excellent test-retest reliability, both within and between laboratory visits and home-based assessments (ICC: 0.79-0.90). These results suggest that dual task walking assessments are valid and can be reliably administered in remote settings in relatively healthy younger and older adults.

\section{ROBOTIC PET THERAPY IMPROVES OUTCOMES FOR HOSPITALIZED OLDER ADULTS}

S. Wexler ${ }^{1}$, L. Drury ${ }^{2}$, C. Pollak ${ }^{1}$, B. Disomma ${ }^{1}$, S. Narducci ${ }^{3}$, S. Iovino ${ }^{3}$, K. Scher ${ }^{3}$, 1. Pace University, 2. Pace University, College of Health Professions, 3. Jamaica Hospital Medical Center

This randomized controlled trial at an inner city community hospital examined the effect of a robotic pet (cat or $\mathrm{dog}$ ) on cognition, loneliness, depression, delirium, falls, restraint use, and 1:1 companion use in 160 adults over age 65 . The study utilized robotic pets which have an audible heartbeat and interact by purring or barking, turning their heads, opening/closing their eyes, snuggling, wagging their tails, moving their paws, and showing their bellies. The average cost of a robotic pet ranged from \$100-\$125. Participants were randomized to one of two groups (80 participants per group). Participants in the experimental group received their choice of a cat or dog robot for the duration of their hospital stay. Since the robotic pets could not be disinfected, the robots were single patient use. Patients were encouraged to take the robotic pet home with them. Participants in the control group received a daily 15 minute visit from a nursing student. Measures (Mini-cog, 3 Item UCLA loneliness scale, GDS, and CAM) were administered upon study enrollment and prior to discharge. Patients who had the robotic pet during hospitalization had less delirium, loneliness, fewer falls, and less 1:1 companion use $(p<0.001)$ than patients who did not have a robot. There was no significant difference in cognition, and depression. Patient and caregiver feedback was overwhelmingly positive. There were too few restraints reported in the sample to allow for statistical modeling. Study findings validate the use of this innovative cost effective technology for hospitalized older adults.

\section{THE EFFECTS OF A THEORY-BASED PATIENT PORTAL ELEARNING PROGRAM FOR OLDER ADULTS WITH CHRONIC ILLNESSES}

E. $\mathrm{Nahm}^{1}$, S. Zhu ${ }^{2}, \mathrm{M}$. Bellantoni ${ }^{3}$, M. Rietschel ${ }^{1}$, V. Russomanno ${ }^{4}, \mathrm{H}$. Son ${ }^{5}$, I. La ${ }^{1}, 1$. University of Maryland School of Nursing, 2. University of Maryland Baltimore, 3. Johns Hopkins University School of Medicine, 4. University of Maryland Medical System, 5. University of Maryland Baltimore, School of Nursing

The high prevalence of chronic illnesses is a serious public health problem in the U.S., and more than 70 million older adults have at least one chronic illness. Although patient portals (PPs), a federally supported health information technology, have been shown to be effective in managing chronic illnesses, low PP adoption rates in older adults were addressed as a particular concern. Lack of support for older adults using PPs remains a critical gap in most implementation processes. Previously, our research team developed and pilot-tested 3-week Theory-based Patient portal eLearning Program (T-PeP) for older adults. The main aim of the study was to assess the impact of T-PeP on PP knowledge, selected health outcomes (self-efficacy for PP use and health decisionmaking and health communication) and PP use in older adults. A two-arm RCT was conducted with older adults $(\mathrm{N}=272)$ who have chronic conditions. Participants were recruited online and data were collected at baseline, 3 weeks, and 4 months. The main intervention effects were tested using linear mixed models. The average age of participants was $70.0+8.5$ years and $78.3 \%(n=213)$ were white. At 3 weeks, the intervention group showed significantly greater improvement than the control group in all outcomes, except PP use. At 4 months, the intervention effects decreased, but PP self-efficacy remained significant $(\mathrm{p}=.006)$ and PP use showed a significant increase in the intervention group $(\mathrm{p}=.029)$. Further studies are needed to test the long-term effects of T-PeP on health behaviors and clinical outcomes using more diverse samples. 